\title{
On the Symbol Error Probability of General Order Rectangular QAM in Nakagami- $m$ Fading
}

\author{
George K. Karagiannidis, Senior Member, IEEE
}

\begin{abstract}
Recently, Beaulieu, following an ingenious concept, presented a closed-form expression for a useful integral, which was used for the evaluation of the symbol error probability (SEP) of general order rectangular quadrature amplitude modulation (QAM) in slow Rayleigh fading. In this letter, these results are extended to Nakagami- $m$ fading channels, deriving a novel closed-form formula for the average over Nakagami- $m$ fading of the product of two Gaussian $Q$-functions, which can be efficiently used to study the impact of fading severity on the error performance of general rectangular QAM constellations.
\end{abstract}

Index Terms-Gaussian $Q$-function, quadrature amplitude modulation (QAM), Nakagami-m fading channels, symbol error probability (SEP).

\section{INTRODUCTION}

G ENERAL order rectangular quadrature amplitude modulation (QAM) constellations can be easily generated from two independent pulse amplitude modulation (PAM) signals ( $M_{I}$-PAM (in-phase) and $M_{Q}$-PAM (quadrature)) and can be also easily demodulated. Although, it is generally a sub-optimal modulation scheme, compared to other $M$-QAM constellations, in the sense that they do not maximally space the constellation points for a given energy, the average transmitted power required to achieve a given minimum distance is only slightly greater than the corresponding one required for the best $M$-QAM scheme. This is the reason that general order rectangular QAM modulation can be efficiently used in practical telecommunications systems [1].

Recently, Beaulieu in [2], presented a novel formula for the symbol error probability (SEP) of general rectangular QAM, in additive white Gaussian noise (AWGN). In the same work, he derived a closed-form expression for the integral

$$
\Upsilon(a, b)=\int_{0}^{\infty} f_{R}(r) Q(r a) Q(r b) d r
$$

where $Q(x)$ is the Gaussian- $Q$ function [3], $a, b$ are real constants and $f_{R}(r)$ is the Rayleigh probability density function (pdf). This integral is involved in several wireless applications (see [2], [3] and the references therein) and was used in [2] to evaluate the average symbol error probability (ASEP) of general rectangular QAM modulations in slow Rayleigh fading.

In this paper, the approach of [2] is extended to Nakagami$m$ fading channels. More specifically, a novel closed-form

Manuscript submitted May 24, 2006. The associate editor coordinating the review of this paper and approving it for publication was Dr. Philippe Ciblat.

G. K. Karagiannidis is with the Dept. of Electrical and Computer Engineering, Aristotle University of Thessaloniki, 54124 Thessaloniki, Greece (e-mail: geokarag@auth.gr).

Digital Object Identifier 10.1109/LCOMM.2006.060798. expression is presented for the average over Nakagami- $m$ fading of the product of two Gaussian $Q$-functions with dissimilar origins. This expression is used to evaluate in closed-form the ASEP of general order rectangular QAM, operating in Nakagami- $m$ fading channels and to present via numerical examples- the impact of fading severity on the system's error performance. Finally, it is shown that the general results, presented in this letter, reduce to some specific cases previously published.

\section{The Average over Nakagami- $m$ FAding of the PRODUCT OF TWO GaUSSIAN $Q$-FUnCTIONS}

Consider the integral $\Upsilon(a, b)$ in (1), but now assuming that $R$ follows Nakagami- $m$ distribution with pdf given by [4]

$$
f_{R}(r)=\frac{2 m^{m}}{\Omega^{m} \Gamma(m)} r^{2 m-1} \exp \left(-\frac{m}{\Omega} r^{2}\right)
$$

where $\Gamma(x)$ is the Gamma function [5, eq. (8.310/1)], $\Omega=$ $\mathcal{E}\left\langle R^{2}\right\rangle, m=\Omega^{2} / \mathcal{E}\left\langle\left(R^{2}-\Omega\right)^{2}\right\rangle \geq 1 / 2$, and $\mathcal{E}\langle\cdot\rangle$ denotes expectation. The PDF in (2) includes the cases of Rayleigh $(m=1)$ and one-sided Gaussian $(m=1 / 2)$ distributions as special ones.

A closed-form solution for (1) for integer values of $m$ and $a=b$, was given by Simon and Alouini in [3, ch. 5.1], while -as mentioned above- for arbitrary values of $a, b$ and $m=1$, Beaulieu presented in [2] a useful closed-form expression as

$$
\begin{aligned}
\Upsilon_{m=1}(a, b) & =\frac{1}{4}-\frac{1}{2 \pi h(a)} \tan ^{-1}\left[\frac{a}{b} h(a)\right] \\
& -\frac{1}{2 \pi h(b)} \tan ^{-1}\left[\frac{b}{a} h(b)\right]
\end{aligned}
$$

with $h(x)=\sqrt{\frac{1+\sigma^{2} x^{2}}{\sigma^{2} x^{2}}}$ and $\sigma^{2}=\frac{\Omega}{2}$. However, to the best of the author' knowledge, closed-form solution to (1) for the general case of Nakagami- $m$ fading channel with dissimilar values for $a, b$, does not exist in the literature.

In Appendix is proved that such a solution is

$$
\Upsilon(a, b)=\frac{1}{4}-[g(a, b)+g(b, a)]
$$

where

$$
\begin{aligned}
& g(a, b)=\frac{1}{\sqrt{\pi}} \sum_{k=0}^{m-1} \frac{2^{k-1} m^{k} a \sqrt{\Omega} \Gamma\left(k+\frac{3}{2}\right)}{\left(a^{2} \Omega+2 m\right)^{k+\frac{1}{2}} k !(1+2 k)} \\
& -\frac{1}{4 \pi} \sum_{k=0}^{m-1} \frac{\Omega a b 2^{k+1} m^{k}{ }_{2} F_{1}\left(1,1+k ; \frac{3}{2} ; \frac{b^{2} \Omega}{a^{2} \Omega+2 m+b^{2} \Omega}\right)}{\left(a^{2} \Omega+2 m+b^{2} \Omega\right)^{k+1}}
\end{aligned}
$$




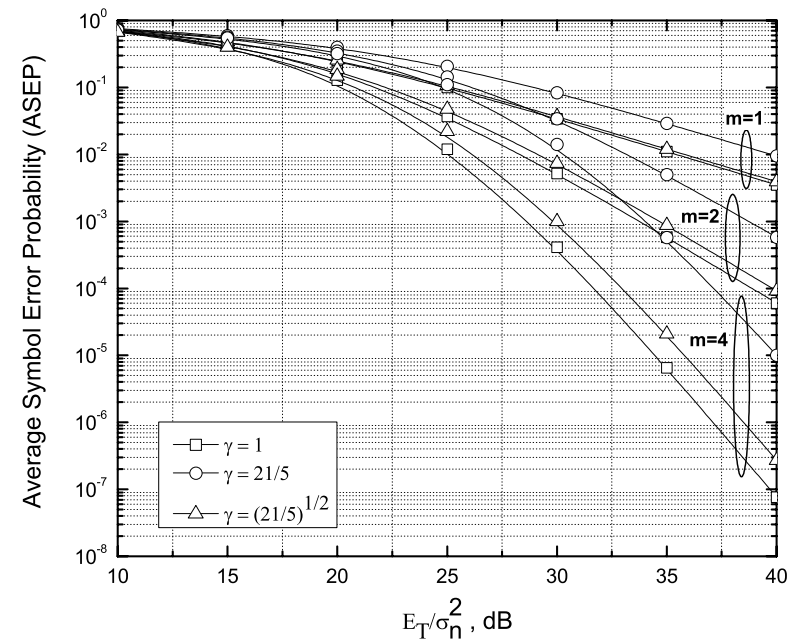

Fig. 1. ASEP for a 8x4-QAM in Nakagami- $m$ fading.

with ${ }_{2} F_{1}(x, y ; z ; w)$ being the Gauss Hypergeometric function [5] and $m$ integer.

For the special case of $m=1$ (Rayleigh fading), using [5, eq. (9.121/14)] and after manipulations, (4) reduces to (3). Also, for $a=b$ and integer $m$, using [5, eq. (9.121/14)], [6] and after laborious manipulations, (4) coincides to the already published result [3, (5.30)].

\section{The IMPACT OF FADING SEVERITY ON THE ERror PERFormance of GENERAL ORDER RECTANGULAR QAM} [2]

The SEP of general order rectangular QAM in AWGN is

$$
\begin{aligned}
P_{S}\left(A_{I}, A_{Q}\right) & =2\left(1-\frac{1}{M_{I}}\right) Q\left(A_{I}\right)+2\left(1-\frac{1}{M_{Q}}\right) Q\left(A_{Q}\right) \\
& -4\left(1-\frac{1}{M_{I}}\right)\left(1-\frac{1}{M_{Q}}\right) Q\left(A_{I}\right) Q\left(A_{Q}\right)
\end{aligned}
$$

where $M_{I}$-PAM and $M_{Q}$-PAM are the in-phase and quadrature PAM signals, $A_{I}=d_{I} / \sigma_{n}$ and $A_{Q}=d_{Q} / \sigma_{n}$, with $d_{I}$, $d_{Q}$ be the decision distances and $\sigma_{n}^{2}$ the noise power.

The ASEP over Nakagami- $m$ fading channels can be written as

$$
P=\int_{0}^{\infty} f_{R}(r) P_{S}\left(r A_{I}, r A_{Q}\right) d r
$$

or

$$
\begin{aligned}
P= & 2\left(1-\frac{1}{M_{I}}\right) \Psi\left(A_{I}\right)+2\left(1-\frac{1}{M_{Q}}\right) \Psi\left(A_{Q}\right) \\
& -4\left(1-\frac{1}{M_{I}}\right)\left(1-\frac{1}{M_{Q}}\right) \Upsilon\left(A_{I}, A_{Q}\right)
\end{aligned}
$$

with

$$
\Psi(a)=\int_{0}^{\infty} Q(a r) f_{R}(r) d r
$$

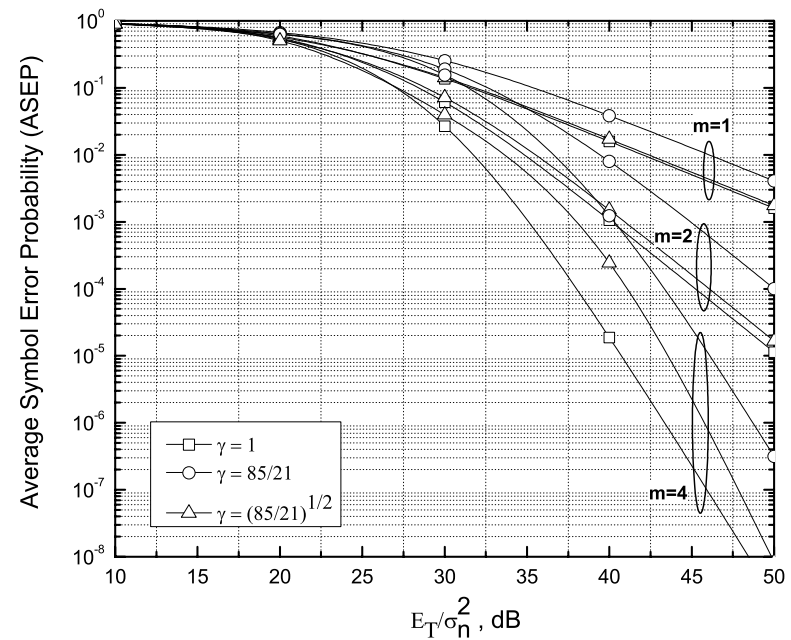

Fig. 2. ASEP for a $16 \times 8$-QAM in Nakagami- $m$ fading.

being the average over Nakagami- $m$ fading of $Q(a x)$. Fortunately, a closed-form solution to $\Psi(a)$ for integer values of $m$ was given in [3, eq. (5.17)] as

$$
\Psi(a)=\frac{1}{2}-\frac{M(a)}{2} \sum_{k=0}^{m-1}\left(\begin{array}{c}
2 k \\
k
\end{array}\right)\left(\frac{1-M(a)^{2}}{4}\right)^{k}
$$

where $M(x)=\sqrt{\frac{x^{2} \Omega}{1+x^{2} \Omega}}$. Substituting (4) and (10) in (8), the ASEP of general order rectangular QAM can be evaluated in closed-form. Next, using the above formulation, we give two examples for different rectangular constellations, to show the impact of fading severity on the system's error performance.

Let us consider a 8x4-QAM system, as in [2]. In Fig. 1 the ASEP is depicted as a function of the average symbol energy-to-noise power, $\frac{E_{T}}{\sigma_{n}^{2}}(d B)$, where the signal-to-noise ratio (SNR) is

$$
\frac{E_{T}}{\sigma_{n}^{2}}=21 \Omega d_{I}^{2}+5 \Omega d_{Q}^{2}=\left(21+5 \gamma^{2}\right) \Omega A_{I}
$$

with $\gamma=\frac{d_{Q}}{d_{I}}=\frac{A_{Q}}{A_{I}}$. It is evident that a decrease in the fading severity (greater $m$ ) significantly affects the system's error performance. A transition from $m=1$ (Rayleigh) to $m=2$ leads to a performance improvement that ranges from 5 to $10 \mathrm{~dB}$ (depending on the $\gamma$ and SNR), while from $m=1$ to $m=4$ this improvement may be greater than 10 $\mathrm{dB}$. Moreover, it is interesting to observe that in low fading environments, the ASEP improvement is higher for the equal decision distance case $(\gamma=1)$ compared to equal average energy $(\gamma=\sqrt{21 / 5})$ in about $0.5 \mathrm{~dB}$ in Rayleigh fading to $2.5 \mathrm{~dB}$ for $m=4$. Also, when the quadrature signal has $21 / 5$ times the average energy of the in-phase signal $(\gamma=21 / 5)$, the ASEP deteriorates substantially in lower fading conditions.

Similar observations can be obtained from Fig. 2, where a $16 \times 8$-QAM is studied. In this case, the SNR is

$$
\frac{E_{T}}{\sigma_{n}^{2}}=85 \Omega d_{I}^{2}+21 \Omega d_{Q}^{2}=\left(85+21 \gamma^{2}\right) \Omega A_{I} .
$$

It is interesting to note here that $\gamma$ has a greater effect to the ASEP compared to 8x4-QAM, especially in higher values of $m$. 


\section{APPENDIX}

Following the concept of $[2$, Appendix $]$, the integral $\Upsilon(a, b)$ in (1) can be splited into two triple integrals, named $\Upsilon_{1}(a, b)$ and $\Upsilon_{2}(a, b)$, as

$$
\begin{aligned}
\Upsilon(a, b)= & \int_{0}^{\infty} \int_{0}^{\frac{b}{a} x_{1}} \int_{0}^{\frac{x_{2}}{b}} f\left(x_{1}\right) f\left(x_{2}\right) f_{R}(r) d r d x_{2} d x_{1} \\
& +\int_{0}^{\infty} \int_{0}^{\frac{a}{b} x_{2}} \int_{0}^{\frac{x_{1}}{a}} f\left(x_{2}\right) f\left(x_{1}\right) f_{R}(r) d r d x_{1} d x_{2} \\
& =\Upsilon_{1}(a, b)+\Upsilon_{1}(b, a)
\end{aligned}
$$

where $f(x)=\frac{e^{-\frac{x^{2}}{2}}}{\sqrt{2 \pi}}$.

The inner integral in $\Upsilon_{1}(a, b)$ is the Nakagami- $m$ cumulative distribution function (cdf) at $\frac{x_{2}}{b}$, which can be solved using [5, eq. (3.381)], resulting in

$$
\begin{aligned}
& \Upsilon_{1}(a, b)=\frac{1}{2 \pi \Gamma(m)} \\
& \int_{0}^{\infty} \int_{0}^{\frac{b}{a} x_{1}} f\left(x_{1}\right) f\left(x_{2}\right)\left[\Gamma(m)-\Gamma\left(m, \frac{m x_{2}^{2}}{b^{2} \Omega}\right)\right] d x_{2} d x_{1}
\end{aligned}
$$

with $\Gamma(x, y)$ being the incomplete Gamma function [5, eq. (8.350.2)]. Substituting $f\left(x_{1}\right)$ and $f\left(x_{2}\right)$ in (14) one has

$\Upsilon_{1}(a, b)=\frac{1}{2 \pi} \int_{0}^{\infty} \int_{0}^{\frac{b}{a} x_{1}} \exp \left(-\frac{x_{1}^{2}}{2}-\frac{x_{2}^{2}}{2}\right) d x_{2} d x_{1}-\frac{1}{2 \pi \Gamma(m)}$ $\int_{0}^{\infty} \int_{0}^{\frac{b}{a} x_{1}} \exp \left(-\frac{x_{1}^{2}}{2}-\frac{x_{2}^{2}}{2}\right) \Gamma\left(m, \frac{m x_{2}^{2}}{b^{2} \Omega}\right) d x_{2} d x_{1}$.

Now, after replacing the incomplete Gamma function with its finite series representation for integer values of $m[5$, $(8.352 / 2)]$

$$
\begin{aligned}
& \Upsilon_{1}(a, b)=\frac{1}{2 \pi} \tan ^{-1}\left(\frac{b}{a}\right)-\frac{(m-1) !}{2 \pi \Gamma(m)} \\
& \int_{0}^{\infty} \int_{0}^{\frac{b}{a} x_{1}} \exp \left(-\frac{x_{1}^{2}+x_{2}^{2}}{2}-\frac{m x_{2}^{2}}{b^{2} \Omega}\right) \sum_{k=0}^{m-1} \frac{\left(\frac{m x_{2}^{2}}{b^{2} \Omega}\right)^{k}}{k !} d x_{2} d x_{1}
\end{aligned}
$$

$$
\begin{aligned}
& =\frac{1}{2 \pi} \tan ^{-1}\left(\frac{b}{a}\right)-\sum_{k=0}^{m-1} \frac{m^{k}}{\left(\frac{b^{2} \Omega+2 m}{2 b^{2} \Omega}\right)^{k+\frac{1}{2}} b^{2 k} \Omega^{k} k !(2+4 k)} \\
& {\left[\sqrt{2 \pi} \Gamma\left(k+\frac{3}{2}\right)-(2 k+1)\right.} \\
& \left.\int_{0}^{\infty} \exp \left(-\frac{x_{1}^{2}}{2}\right) \Gamma\left(k+\frac{1}{2}, \frac{b^{2}\left(\frac{b^{2} \Omega+2 m}{2 b^{2} \Omega}\right) x_{1}^{2}}{a^{2}}\right) d x_{1}\right] .
\end{aligned}
$$

The integral in (17) can be solved using [5, eq. (6.455)] resulting after manipulations in

$$
\begin{aligned}
\Upsilon_{1}(a, b)= & \frac{1}{2 \pi} \tan ^{-1}\left(\frac{b}{a}\right) \\
- & \frac{1}{\sqrt{\pi}} \sum_{k=0}^{m-1} \frac{2^{k-1} m^{k} b \sqrt{\Omega} \Gamma\left(k+\frac{3}{2}\right)}{\left(b^{2} \Omega+2 m\right)^{k+\frac{1}{2}} k !(1+2 k)} \\
& +\frac{1}{4 \pi} \sum_{k=0}^{m-1} \frac{\Omega b a 2^{k+1} m^{k}}{\left(b^{2} \Omega+2 m+a^{2} \Omega\right)^{k+1}} \\
& { }_{2} F_{1}\left(1,1+k ; \frac{3}{2} ; \frac{a^{2} \Omega}{b^{2} \Omega+2 m+a^{2} \Omega}\right) .
\end{aligned}
$$

Now, using (13), (4) is proved.

\section{ACKNOWLEDGMENT}

The author would like to thank A. Lioumpas for preparing the figures of this paper

\section{REFERENCES}

[1] J. G. Proakis, Digital Communications, Fourth Edition. New York: McGraw-Hill, 2001.

[2] N. C. Beaulieu, "A useful integral for wireless communication theory and its application to rectangular signaling constellation error rates," IEEE Trans. Commun., vol. 54, no. 5, pp. 802-805, May 2006.

[3] M. K. Simon and M.-S. Alouini, Digital Communication over Fading Channels, Second Edition. New York: Wiley, 2005.

[4] M. Nakagami, "The $m$-distribution: a general formula of intensity distribution of rapid fading," Statistical Methods in Radio Wave Propagation, vol. 40, pp. 757-768, Nov. 1962.

[5] I. S. Gradshteyn and I. M. Ryzhik, Table of Integrals, Series, and Products, Fifth Edition. New York: Academic, 1994.

[6] Wolfram. (2006) The Wolfram functions site. Internet. [Online]. Available: http://functions.wolfram.com/07.23.03.0177.01 